\author{
S.M. Davoodi*, N.A. Abdul Rahman \\ School of Mathematical Sciences, Universiti Sains Malaysia, Penang, Malaysia \\ (E-mail: smd1376@gmail.com,aswad.rahman@usm.my)
}

\title{
Predicting the optimal solution in fuzzy linear programming problem
}

\begin{abstract}
In this paper we try to define a percentage form of LR fuzzy numbers which is a useful form of fuzzy numbers and its' arithmetics. So, we show how the maximum variation range of optimal value of fuzzy objective function can be predicted by using this form of fuzzy numbers. Since fuzzy problems are generally solved through a complicated manner, the purpose of this study is releasing a kind of prediction for the final solution in the way that the manager can access to an outlook to optimal solution $\left(Z^{*}\right)$ without solving the problem. Finally, optimal value of fuzzy objective function on fuzzy linear programming is predicted when maximum variation range of fuzzy variable have been predetermined.
\end{abstract}

Keywords: Percentage form of fuzzy numbers, Fuzzy number, Fuzzy linear programming, Fuzzy Arithmetic

\section{Introduction}

In the recent past the fields of management science, operational research and industrial engineering have dedicated a lot of attention to decision making theories, decision making methods and application. For the past sixty years, many research works in these areas have been documented. However, there is a need to develop new methods that would fit real world problems in the context of linear programming. The traditional methods of linear programming modeling require precise model parameters and this, is not obtainable in real world problems. So, researchers usually estimate model parameters by themselves and These estimated values of the model parameters may not be precise.

Tanaka et al. [1] proposed the concept of fuzzy mathematical programming based on the fuzzy decision framework of Bellman and Zadeh [2]. Zimmerman [3] introduced the first formulation of fuzzy linear programming (FLP) to address the impreciseness of the parameters in linear programming (LP) problems with fuzzy constraints and objective functions. A number of researchers have exhibited their interest to solve the FLP problems [4-13] and fully FLP problems [14-23].

A new form of fuzzy numbers called percentage form of LR fuzzy numbers (PLR fuzzy number) is now introduced in this paper. This form of fuzzy numbers are applied to many discipline such as industrial application, mathematical modeling and management sciences. Arithmetic operations of this form of fuzzy numbers and some properties of it is studied. Also the prediction process of fuzzy optimal solution and its variation range is described in this paper according to properties of this form of fuzzy numbers.

The solution of FLP problems and fully FLP problems cannot be calculated by applying the majority of existing methods in which some or all the parameters are represented by unrestricted LR fuzzy numbers. In addition, there are large computational procedure with some existing methods [20]. By predicting the range of fuzzy numbers' change and reaching to an outlook of optimal solution and also having an alternating fuzzy variables' frequency range on demand, we are able to find the optimal solution with ideal frequency range.

\section{Preliminary Concepts}

In this section, we introduce some basic definitions on fuzzy set theory and the main concepts needed in the paper.

Definition 1. Let $X$ be a collection of objects denoted generically by $x$. $\widetilde{A}$ is called $a$ fuzzy set in $X$ if $\widetilde{A}$ is a set of ordered pairs:

$$
\widetilde{A}=\left\{\left(x, \mu_{\widetilde{A}}(x)\right) \mid x \in X\right\}
$$

where $\mu_{\widetilde{A}}(x)$ is a membership function of $x$ in $\mu_{\widetilde{A}}$ such that $\mu_{\widetilde{A}}(x): X \rightarrow[0,1]$.

\footnotetext{
${ }^{*}$ Corresponding author.

E-mail:smd1376@gmail.com
} 
Definition 2. A fuzzy number $\widetilde{A}$ is said to be a $L R$-flat fuzzy number, $\widetilde{A}=\left(a^{l}, a^{u}, \alpha, \beta\right)_{L R}$, if its membership function is defined as

$$
\mu_{\widetilde{A}}(x)= \begin{cases}L\left(\frac{a^{l}-x}{\alpha}\right) & , \quad x<a^{l} \\ 1 & , \quad a^{l} \leq x \leq a^{u} \\ R\left(\frac{x-a^{u}}{\beta}\right) & , \quad x>a^{u} \\ 0 & , \text { otherwise }\end{cases}
$$

where $L$ and $R$ are reference functions, i.e., $L, R:[0,+\infty) \rightarrow[0,1]$ are non-increasing that $L(0)=R(0)=$ 1 and $L(1)=R(1)=0$. The membership function of a $L R$ fuzzy number, $\widetilde{A}=(a, \alpha, \beta)_{L R}$, can be also defined as

$$
\mu_{\widetilde{A}}(x)= \begin{cases}L\left(\frac{a-x}{\alpha}\right) & , \quad x \leq a \\ R\left(\frac{x-a}{\beta}\right), & x>a \\ 0 & , \text { otherwise }\end{cases}
$$

Definition 3. Let $\widetilde{A}_{1}=\left(a_{1}, \alpha_{1}, \beta_{1}\right)_{L R}$ and $\widetilde{A}_{2}=\left(a_{2}, \alpha_{2}, \beta_{2}\right)_{L R}$ be $L R$ fuzzy numbers and $r \in \mathbb{R},(r \neq 0)$ then arithmetic on fuzzy numbers are defined

$$
\begin{aligned}
& \widetilde{A}_{1} \oplus \widetilde{A}_{2}=\left(a_{1}+a_{2}, \alpha_{1}+\alpha_{2}, \beta_{1}+\beta_{2}\right)_{L R} \\
& r \widetilde{A}_{1}= \begin{cases}\left(r a_{1}, r \alpha_{1}, r \beta_{1}\right)_{L R} & , \quad r>0 \\
\left(r a_{1},-r \beta_{1},-r \alpha_{1}\right)_{L R} & , \quad r<0\end{cases} \\
& \widetilde{A}_{1} \otimes \widetilde{A}_{2}=\left\{\begin{array}{rlll}
\left(a_{1} a_{2}, a_{1} \alpha_{2}+a_{2} \alpha_{1}, a_{1} \beta_{2}+a_{2} \beta_{1}\right)_{L R} & , \text { where } \widetilde{A}_{1} \succ 0 & , \widetilde{A}_{2} \succ 0 \\
\left(a_{1} a_{2}, a_{2} \alpha_{1}-a_{1} \beta_{2}, a_{2} \beta_{1}-a_{1} \alpha_{2}\right)_{L R} & , \text { where } \widetilde{A}_{1} \prec 0 & , \widetilde{A}_{2} \succ 0 \\
\left(a_{1} a_{2}, a_{1} \alpha_{2}-a_{2} \beta_{1}, a_{1} \beta_{2}-a_{2} \alpha_{1}\right)_{L R} & , \text { where } \widetilde{A}_{1} \succ 0 & , \widetilde{A}_{2} \prec 0 \\
\left(a_{1} a_{2},-a_{1} \beta_{2}-a_{2} \beta_{1},-a_{1} \alpha_{2}-a_{2} \alpha_{1}\right)_{L R} & , \text { where } \widetilde{A}_{1} \prec 0 & , & \widetilde{A}_{2} \prec 0
\end{array}\right.
\end{aligned}
$$

Remark 1 . We denote the set of all $L R$-fuzzy numbers by $\mathcal{F}(\mathbb{R})$.

Definition 4. A linear ranking function is a function $\mathfrak{R}: \mathcal{F}(\mathbb{R}) \rightarrow \mathbb{R}$, which maps each fuzzy number into a real line, where there is a natural order.

$$
\Re(\widetilde{A})=\frac{1}{2} \int_{0}^{1}\left(L_{h}(x)+R_{h}(x)\right) d h
$$

where $\left[L_{h}(x), R_{h}(x)\right]$ is $h$ - cute of $\widetilde{A}$.

Let $\widetilde{A}=(a, \alpha, \beta)$ be a triangular fuzzy number then

$$
\mathfrak{R}(\widetilde{A})=a+\frac{1}{4}(\beta-\alpha) .
$$

\section{PLR fuzzy number}

Here a very useful form of fuzzy numbers is discussed and the arithmetic definition of this form of fuzzy numbers is given. We also show that the arithmetic of this form of fuzzy numbers is simple when compared with the current form of fuzzy numbers.

Definition 5. (PLR fuzzy number) Let

$$
\widetilde{A}=(a, \alpha, \beta)_{L R}, \quad \alpha, \beta \geq 0 \text { and } a \neq 0
$$

be the $L R$ fuzzy number, then

$$
\widetilde{A}=(a, \widehat{\alpha} \%, \widehat{\beta} \%), \quad \widehat{\alpha}, \widehat{\beta} \geq 0 \text { and } a \neq 0
$$


is called $P L R$ fuzzy number and the $\widehat{\alpha}$ and $\widehat{\beta}$ are the left and right percentage deviation from the center of fuzzy numbers $(a)$, respectively. The formula to convert $L R$ fuzzy number to $P L R$ fuzzy numbers and back are as follows :

$$
\begin{aligned}
& \widetilde{A}=(a, \alpha, \beta)_{L R}=\left(a, \frac{\alpha}{|a|} 100 \%, \frac{\beta}{|a|} 100 \%\right), \\
& \widetilde{A}=(a, \alpha \%, \beta \%)=\left(a, \frac{\alpha|a|}{100}, \frac{\beta|a|}{100}\right)_{L R} .
\end{aligned}
$$

Note that, $L R$ fuzzy numbers and $P L R$ fuzzy number are called normal and percentage form of fuzzy numbers respectively.

Example 1. Suppose $\widetilde{A}=(10,2,3)_{L R}$ is a $L R$ fuzzy number then its $P L R$ form is as follow:

$$
\widetilde{A}=(10,2,3)_{L R}=\left(10, \frac{2}{|10|} 100 \%, \frac{3}{|10|} 100 \%\right)=(10,20 \%, 30 \%) .
$$

Furthermore suppose $\widetilde{A}=(-50,8 \%, 14 \%)$ is a $P L R$ fuzzy number then its $L R$ form is as follow:

$$
\widetilde{A}=(-50,8 \%, 14 \%)=\left(-50, \frac{8 \times|-50|}{100}, \frac{14 \times|-50|}{100}\right)_{L R}=(-50,4,7)_{L R} .
$$

Definition 6. Suppose $\widetilde{A}=(a, \alpha \%, \beta \%)$ is $P L R$ fuzzy number, if $\alpha=\beta$ then $\widetilde{A}$ is symmetric $P L R$ fuzzy number and we show $\widetilde{A}=(a, \alpha \%)$.

\section{Arithmetic Operation on PLR Fuzzy Numbers}

Let $\widetilde{A}_{1}=\left(a_{1}, \alpha_{1} \%, \beta_{1} \%\right)$ and $\widetilde{A}_{2}=\left(a_{2}, \alpha_{2} \%, \beta_{2} \%\right)$ denote $P L R$ fuzzy numbers and $r \in \mathbb{R},(r \neq 0)$ then $L R$ form of $\widetilde{A}_{1}$ and $\widetilde{A}_{2}$ are as follows:

$$
\widetilde{A}_{1}=\left(a_{1}, \frac{\alpha_{1}\left|a_{1}\right|}{100}, \frac{\beta_{1}\left|a_{1}\right|}{100}\right)_{L R}, \quad \widetilde{A}_{2}=\left(a_{2}, \frac{\alpha_{2}\left|a_{2}\right|}{100}, \frac{\beta_{2}\left|a_{2}\right|}{100}\right)_{L R} .
$$

Addition $(\oplus)$ : According to Equation (1),we obtain

$$
\begin{aligned}
\widetilde{A}_{1} \oplus \widetilde{A}_{2} & =\left(a_{1}+a_{2}, \frac{\alpha_{1}\left|a_{1}\right|}{100}+\frac{\alpha_{2}\left|a_{2}\right|}{100}, \frac{\beta_{1}\left|a_{1}\right|}{100}+\frac{\beta_{2}\left|a_{2}\right|}{100}\right)_{L R} \\
& =\left(a_{1}+a_{2}, \frac{\alpha_{1}\left|a_{1}\right|+\alpha_{2}\left|a_{2}\right|}{100}, \frac{\beta_{1}\left|a_{1}\right|+\beta_{2}\left|a_{2}\right|}{100}\right)_{L R} .
\end{aligned}
$$

$P L R$ form of above $L R$ fuzzy number is as follow:

$$
\begin{aligned}
\widetilde{A}_{1} \oplus \widetilde{A}_{2} & =\left(a_{1}+a_{2}, \frac{\alpha_{1}\left|a_{1}\right|+\alpha_{2}\left|a_{2}\right|}{100} \times \frac{100 \%}{\left|a_{1}+a_{2}\right|}, \frac{\beta_{1}\left|a_{1}\right|+\beta_{2}\left|a_{2}\right|}{100} \times \frac{100 \%}{\left|a_{1}+a_{2}\right|}\right) \\
& =\left(a_{1}+a_{2}, \frac{\alpha_{1}\left|a_{1}\right|+\alpha_{2}\left|a_{2}\right|}{\left|a_{1}+a_{2}\right|} \%, \frac{\beta_{1}\left|a_{1}\right|+\beta_{2}\left|a_{2}\right|}{\left|a_{1}+a_{2}\right|} \%\right) .
\end{aligned}
$$

Scalar Multiplication:

Case 1: If $r>0$ as a result $|r a|=r|a|$ therefore

$$
r \widetilde{A}=\left(r a, r \frac{\alpha|a|}{100}, r \frac{\beta|a|}{100}\right)_{L R} .
$$

$P L R$ form of above $L R$ fuzzy number is as follow:

$$
\begin{aligned}
r \widetilde{A} & =\left(r a, r \frac{\alpha|a|}{100} \times \frac{100 \%}{|r a|}, \frac{r \beta|a|}{100} \times \frac{100 \%}{|r a|}\right) \\
& =\left(r a, r \frac{\alpha|a|}{100} \times \frac{100 \%}{r|a|}, \frac{r \beta|a|}{100} \times \frac{100 \%}{r|a|}\right)=(r a, \alpha \%, \beta \%) .
\end{aligned}
$$

Case 2: If $r<0$, similar to Case 1 we have

$$
r \widetilde{A}=(r a, \beta \%, \alpha \%) .
$$




\section{Multiplication:}

Case 1: Let $\widetilde{A}_{1}$ and $\widetilde{A}_{2}$ be non-negative $P L R$ fuzzy numbers then $\left|a_{1}\right|=a_{1},\left|a_{2}\right|=a_{2}$ and $\left|a_{1} a_{2}\right|=a_{1} a_{2}$. According to Equations (2), we have

$$
\begin{aligned}
\widetilde{A}_{1} \otimes \widetilde{A}_{2} & =\left(a_{1} a_{2}, \frac{\alpha_{1} a_{1}}{100} a_{2}+\frac{\alpha_{2} a_{2}}{100} a_{1}, \frac{\beta_{1} a_{1}}{100} a_{2}+\frac{\beta_{2} a_{2}}{100} a_{1}\right)_{L R} \\
& =\left(a_{1} a_{2}, \frac{a_{1} a_{2}\left(\alpha_{1}+\alpha_{2}\right)}{100}, \frac{a_{1} a_{2}\left(\beta_{1}+\beta_{2}\right)}{100}\right)_{L R} .
\end{aligned}
$$

$P L R$ form of above $L R$ fuzzy number is as follow:

$$
\begin{aligned}
\widetilde{A}_{1} \otimes \widetilde{A}_{2} & =\left(a_{1} a_{2}, \frac{a_{1} a_{2}\left(\alpha_{1}+\alpha_{2}\right)}{100} \times \frac{100}{\left|a_{1} a_{2}\right|} \%, \frac{a_{1} a_{2}\left(\beta_{1}+\beta_{2}\right)}{100} \times \frac{100}{\left|a_{1} a_{2}\right|} \%\right) \\
\Rightarrow \widetilde{A}_{1} \otimes \widetilde{A}_{2} & =\left(a_{1} a_{2},\left(\alpha_{1}+\alpha_{2}\right) \%,\left(\beta_{1}+\beta_{2}\right) \%\right) .
\end{aligned}
$$

Case 2: Let $\widetilde{A}_{1}=\left(a_{1}, \alpha_{1} \%, \beta_{1} \%\right)$ and $\widetilde{A}_{2}=\left(a_{2}, \alpha_{2} \%, \beta_{2} \%\right)$ be non-positive $P L R$ fuzzy numbers then

$$
\widetilde{A}_{1} \otimes \widetilde{A}_{2}=\left(a_{1} a_{2},\left(\beta_{1}+\beta_{2}\right) \%,\left(\alpha_{1}+\alpha_{2}\right) \%\right) .
$$

Case 3: Let $\widetilde{A}_{1}=\left(a_{1}, \alpha_{1} \%, \beta_{1} \%\right)$ is non-negative and $\widetilde{A}_{2}=\left(a_{2}, \alpha_{2} \%, \beta_{2} \%\right)$ be non-positive PLR fuzzy numbers then

$$
\widetilde{A}_{1} \otimes \widetilde{A}_{2}=\left(a_{1} a_{2},\left(\alpha_{2}+\beta_{1}\right) \%,\left(\alpha_{1}+\beta_{2}\right) \%\right) .
$$

Case 4: Let $\widetilde{A}_{1}=\left(a_{1}, \alpha_{1} \%, \beta_{1} \%\right)$ is non-positive and $\widetilde{A}_{2}=\left(a_{2}, \alpha_{2} \%, \beta_{2} \%\right)$ be non-negative PLR fuzzy numbers then

$$
\widetilde{A}_{1} \otimes \widetilde{A}_{2}=\left(a_{1} a_{2},\left(\alpha_{1}+\beta_{2}\right) \%,\left(\alpha_{2}+\beta_{1}\right) \%\right)
$$

In summing up, if $\widetilde{A}_{1}=\left(a_{1}, \alpha_{1} \%, \beta_{1} \%\right)$ and $\widetilde{A}_{2}=\left(a_{2}, \alpha_{2} \%, \beta_{2} \%\right)$ be $P L R$ fuzzy numbers then

$$
\begin{aligned}
& \widetilde{A}_{1} \oplus \widetilde{A}_{2}=\left(a_{1}+a_{2}, \frac{\alpha_{1}\left|a_{1}\right|+\alpha_{2}\left|a_{2}\right|}{\left|a_{1}+a_{2}\right|} \%, \frac{\beta_{1}\left|a_{1}\right|+\beta_{2}\left|a_{2}\right|}{\left|a_{1}+a_{2}\right|} \%\right) \\
& r \widetilde{A}= \begin{cases}(r a, \alpha \%, \beta \%) & , \quad r>0 \\
(r a, \beta \%, \alpha \%) & , \quad r<0\end{cases} \\
& \widetilde{A}_{1} \otimes \widetilde{A}_{2}=\left\{\begin{array}{lll}
\left(a_{1} a_{2},\left(\alpha_{1}+\alpha_{2}\right) \%,\left(\beta_{1}+\beta_{2}\right) \%\right) & , \widetilde{A}_{1} \succeq 0, & \widetilde{A}_{2} \succeq 0 \\
\left(a_{1} a_{2},\left(\alpha_{1}+\beta_{2}\right) \%,\left(\alpha_{2}+\beta_{1}\right) \%\right) & , \widetilde{A}_{1} \preceq 0, & \widetilde{A}_{2} \succeq 0 \\
\left(a_{1} a_{2},\left(\alpha_{2}+\beta_{1}\right) \%,\left(\alpha_{1}+\beta_{2}\right) \%\right) & , \widetilde{A}_{1} \succeq 0, & \widetilde{A}_{2} \preceq 0 \\
\left(a_{1} a_{2},\left(\beta_{1}+\beta_{2}\right) \%,\left(\alpha_{1}+\alpha_{2}\right) \%\right) & , \widetilde{A}_{1} \preceq 0, & \widetilde{A}_{2} \preceq 0
\end{array}\right.
\end{aligned}
$$

Proposition 1. Let $\widetilde{A}_{1}=\left(a_{1}, \alpha_{1} \%, \beta_{1} \%\right), \widetilde{A}_{2}=\left(a_{2}, \alpha_{2} \%, \beta_{2} \%\right), \ldots, \widetilde{A}_{n}=\left(a_{n}, \alpha_{n} \%, \beta_{n} \%\right)$ be PLR fuzzy numbers and

$$
\begin{aligned}
& P=\left\{i \mid \widetilde{A}_{i} \succ 0, \quad i=1,2, \ldots, n\right\} \\
& N=\left\{i \mid \widetilde{A}_{i} \prec 0, \quad i=1,2, \ldots, n\right\}
\end{aligned}
$$

then

$$
\widetilde{A}_{1} \otimes \widetilde{A}_{2} \otimes \cdots \otimes \widetilde{A}_{n}=\left(a_{1} a_{2} \cdots a_{n},\left(\sum_{i \in P} \alpha_{i}+\sum_{i \in N} \beta_{i}\right) \%,\left(\sum_{i \in N} \alpha_{i}+\sum_{i \in P} \beta_{i}\right) \%\right)
$$


Proposition 2. Let $\widetilde{A}_{1}=\left(a_{1}, \alpha_{1} \%, \beta_{1} \%\right), \widetilde{A}_{2}=\left(a_{2}, \alpha_{2} \%, \beta_{2} \%\right), \ldots, \widetilde{A}_{n}=\left(a_{n}, \alpha_{n} \%, \beta_{n} \%\right)$ be non-negative $P L R$ fuzzy numbers then

$$
\widetilde{A}_{1} \otimes \widetilde{A}_{2} \otimes \cdots \otimes \widetilde{A}_{n}=\left(a_{1} a_{2} \cdots a_{n},\left(\alpha_{1}+\alpha_{2}+\cdots+\alpha_{n}\right) \%,\left(\beta_{1}+\beta_{2}+\cdots+\beta_{n}\right) \%\right) .
$$

Proposition 3. Let $\widetilde{A}_{1}=\left(a_{1}, \alpha_{1} \%, \beta_{1} \%\right), \widetilde{A}_{2}=\left(a_{2}, \alpha_{2} \%, \beta_{2} \%\right), \ldots, \widetilde{A}_{n}=\left(a_{n}, \alpha_{n} \%, \beta_{n} \%\right)$ be non-positive $P L R$ fuzzy numbers then

$$
\widetilde{A}_{1} \otimes \widetilde{A}_{2} \otimes \cdots \otimes \widetilde{A}_{n}=\left(a_{1} a_{2} \cdots a_{n},\left(\beta_{1}+\beta_{2}+\cdots+\beta_{n}\right) \%,\left(\alpha_{1}+\alpha_{2}+\cdots+\alpha_{n}\right) \%\right) .
$$

Example 2. Let $\widetilde{A}_{1}=(4,2 \%, 3 \%), \widetilde{A}_{2}=(-5,8 \%, 4 \%), \widetilde{A}_{3}=(6,9 \%, 5 \%), \widetilde{A}_{4}=(3,2 \%, 8 \%)$ and $\widetilde{A}_{5}=(-3,1 \%, 6 \%)$ be $P L R$ fuzzy numbers. Then $P=\left\{i \mid \widetilde{A}_{i} \succ 0\right\}=\{1,3,4\}$ and $N=\left\{i \mid \widetilde{A}_{i} \prec 0\right\}=$ $=\{2,5\}$. Multiplying these fuzzy numbers is calculated as follows:

$$
\begin{aligned}
& \widetilde{A}_{1} \otimes \widetilde{A}_{2} \otimes \widetilde{A}_{3} \otimes \widetilde{A}_{4} \otimes \widetilde{A}_{5} \\
& =\left(4 \times(-5) \times 6 \times 3 \times(-3),\left[\sum_{i \in\{1,3,4\}} \alpha_{i}+\sum_{i \in\{2,5\}} \beta_{i}\right] \%,\left[\sum_{i \in\{2,5\}} \alpha_{i}+\sum_{i \in\{1,3,4\}} \beta_{i}\right] \%\right) \\
& =(1080,[(2+9+2)+(4+6)] \%,[(8+1)+(3+5+8)] \%) \\
& =(1080,23 \%, 25 \%) .
\end{aligned}
$$

According to Proposition 1, it is observed that multiplication of fuzzy numbers by $P L R$ fuzzy numbers is simple and uncomplicated in contrast to $L R$ fuzzy numbers.

\section{Predicting fuzzy optimal solution}

In this section, we describe how the maximum variation range of optimal solution $\left(\widetilde{Z}^{*}\right)$ can be predicted respectively for general form of fuzzy linear programming and special form of fuzzy linear programming problem when the maximum variation range of fuzzy variable is predetermined.

Proposition 4. Let $\widetilde{A}_{1}=\left(a_{1}, \alpha_{1} \%, \beta_{1} \%\right)$ and $\widetilde{A}_{2}=\left(a_{2}, \alpha_{2} \%, \beta_{2} \%\right)$ be $P L R$ fuzzy numbers and $a_{1} a_{2}>0$ then

$$
\widetilde{A}_{1} \oplus \widetilde{A}_{2}=(\widehat{a}, \widehat{\alpha} \%, \widehat{\beta} \%)
$$

then

$$
\begin{aligned}
& \min \left\{\alpha_{1}, \alpha_{2}\right\} \leq \widehat{\alpha} \leq \max \left\{\alpha_{1}, \alpha_{2}\right\} \\
& \min \left\{\beta_{1}, \beta_{2}\right\} \leq \widehat{\beta} \leq \max \left\{\beta_{1}, \beta_{2}\right\}
\end{aligned}
$$

Proof. Without prejudice to the generality of the problem suppose $\alpha_{1} \leq \alpha_{2}$ as a result $\max \left\{\alpha_{1}, \alpha_{2}\right\}=\alpha_{2}$ and $\min \left\{\alpha_{1}, \alpha_{2}\right\}=\alpha_{1}$. In addition,

$$
a_{1} a_{2}>0 \Rightarrow\left|a_{1}+a_{2}\right|=\left|a_{1}\right|+\left|a_{2}\right| \neq 0
$$

then

$$
\begin{aligned}
& \alpha_{1} \leq \alpha_{2} \\
\Rightarrow & \alpha_{1}\left|a_{1}\right| \leq \alpha_{2}\left|a_{1}\right| \\
\Rightarrow & \alpha_{1}\left|a_{1}\right|+\alpha_{2}\left|a_{2}\right| \leq \alpha_{2}\left|a_{1}\right|+\alpha_{2}\left|a_{2}\right| \\
\Rightarrow & \alpha_{1}\left|a_{1}\right|+\alpha_{2}\left|a_{2}\right| \leq \alpha_{2}\left(\left|a_{1}\right|+\left|a_{2}\right|\right)=\alpha_{2}\left|a_{1}+a_{2}\right| \\
\Rightarrow & \frac{\alpha_{1} a_{1}+\alpha_{2} a_{2}}{\left|a_{1}+a_{2}\right|} \leq \alpha_{2}=\max \left\{\alpha_{1}, \alpha_{2}\right\} \\
\Rightarrow & \widehat{\alpha} \leq \max \left\{\alpha_{1}, \alpha_{2}\right\}
\end{aligned}
$$




$$
\begin{aligned}
& \alpha_{2} \geq \alpha_{1} \\
\Rightarrow & \alpha_{2}\left|a_{2}\right| \geq \alpha_{1}\left|a_{2}\right| \\
\Rightarrow & \alpha_{1}\left|a_{1}\right|+\alpha_{2}\left|a_{2}\right| \geq \alpha_{1}\left|a_{1}\right|+\alpha_{1}\left|a_{2}\right| \\
\Rightarrow & \alpha_{1}\left|a_{1}\right|+\alpha_{2}\left|a_{2}\right| \geq \alpha_{1}\left(\left|a_{1}\right|+\left|a_{2}\right|\right)=\alpha_{1}\left|a_{1}+a_{2}\right| \\
\Rightarrow & \frac{\alpha_{1} a_{1}+\alpha_{2} a_{2}}{\left|a_{1}+a_{2}\right|} \geq \alpha_{1}=\min \left\{\alpha_{1}, \alpha_{2}\right\} \\
\Rightarrow & \widehat{\alpha} \geq \min \left\{\alpha_{1}, \alpha_{2}\right\}
\end{aligned}
$$

$$
\text { (3) and (4) } \Rightarrow \min \left\{\alpha_{1}, \alpha_{2}\right\} \leq \widehat{\alpha} \leq \max \left\{\alpha_{1}, \alpha_{2}\right\} \text {. }
$$

Proof for $\widehat{\beta}$ is similar to the proof for $\widehat{\alpha}$.

Proposition 5. Let $\widetilde{A}_{1}=\left(a_{1}, \alpha_{1} \%, \beta_{1} \%\right), \widetilde{A}_{2}=\left(a_{2}, \alpha_{2} \%, \beta_{2} \%\right), \ldots, \widetilde{A}_{n}=\left(a_{n}, \alpha_{n} \%, \beta_{n} \%\right)$ be PLR fuzzy numbers and $a_{i} a_{j}>0, i \neq j$ then

$$
\widetilde{A}_{1} \oplus \widetilde{A}_{2} \oplus \cdots \oplus \widetilde{A}_{n}=\left(a_{1}+a_{2}+\cdots+a_{n}, \widehat{\alpha} \%, \widehat{\beta} \%\right)
$$

then

$$
\min \left\{\alpha_{1}, \alpha_{2}, \ldots, \alpha_{n}\right\} \leq \widehat{\alpha} \leq \max \left\{\alpha_{1}, \alpha_{2}, \ldots, \alpha_{n}\right\}
$$

and

$$
\min \left\{\beta_{1}, \beta_{2}, \ldots, \beta_{n}\right\} \leq \widehat{\beta} \leq \max \left\{\beta_{1}, \beta_{2}, \ldots, \beta_{n}\right\}
$$

Predicting maximum variation range of objective function

Now we show how the maximum variation range of fuzzy objective function is predicted for general form of fully fuzzy linear programming problems.

Let a fully fuzzy linear programming be defined as follow:

$$
\begin{aligned}
& \begin{array}{l}
\text { Min } \\
\operatorname{Max}
\end{array} \quad \widetilde{Z}=\sum_{j=1}^{n} \widetilde{c}_{j} \otimes \widetilde{x}_{j} \\
& \text { s.t. } \quad \sum_{j=1}^{n} \widetilde{a}_{i j} \otimes \widetilde{x}_{j} \begin{array}{l}
\underset{\text { or }}{\approx} \\
\text { or }
\end{array} \quad \widetilde{b}_{i} \quad, \quad i=1,2, \ldots, m \\
& \widetilde{x}_{j} \succeq 0
\end{aligned}
$$

where $\widetilde{x}_{j}=\left(x_{j}, \alpha_{x_{j}} \%, \beta_{x_{j}} \%\right), \widetilde{c}_{j}=\left(c_{j}, \alpha_{c_{j}} \%, \beta_{c_{j}} \%\right), \widetilde{a}_{i j}=\left(a_{i j}, \alpha_{a_{i j}} \%, \beta_{a_{i j}} \%\right)$ and $\widetilde{b}_{i}=\left(b_{i}, \alpha_{b_{i}} \%, \beta_{b_{i}} \%\right)$ be $P L R$ fuzzy numbers for $i=1, \ldots, m, j=1, \ldots, n$.

Theorem 1. Let $\widetilde{c}_{j}=\left(c_{j}, \alpha_{c_{j}} \%, \beta_{c_{j}} \%\right)$ and $\widetilde{x}_{j}=\left(x_{j}, \alpha_{x_{j}} \%, \beta_{x_{j}} \%\right), j=1,2, \ldots, n$ be non-negative $P L R$ fuzzy numbers and $\widetilde{x}_{j}$ be feasible solution of fully fuzzy linear programming problem (5) also

$$
\widetilde{Z}=(\widehat{z}, \widehat{\alpha} \%, \widehat{\beta} \%)=\sum_{j=1}^{n} \widetilde{c}_{j} \otimes \widetilde{x}_{j}
$$

is the objective function of fully fuzzy linear programming problem (32) then

$$
\widehat{z}=c_{1} x_{1}+c_{2} x_{2}+\cdots+c_{n} x_{n}
$$

and

$$
\alpha_{\min } \leq \widehat{\alpha} \leq \alpha_{\max }, \quad \beta_{\min } \leq \widehat{\beta} \leq \beta_{\max }
$$


where

$$
\begin{aligned}
& \alpha_{\text {min }}=\min \left\{\alpha_{c_{i}}+\alpha_{x_{i}} \mid i=1,2, \ldots, n\right\} \\
& \alpha_{\text {max }}=\max \left\{\alpha_{c_{i}}+\alpha_{x_{i}} \mid i=1,2, \ldots, n\right\} \\
& \beta_{\text {min }}=\min \left\{\beta_{c_{i}}+\beta_{x_{i}} \mid i=1,2, \ldots, n\right\} \\
& \beta_{\text {max }}=\max \left\{\beta_{c_{i}}+\beta_{x_{i}} \mid i=1,2, \ldots, n\right\}
\end{aligned}
$$

Theorem 2. Let $\widetilde{c}_{j}=\left(c_{j}, \alpha_{c_{j}} \%, \beta_{c_{j}} \%\right)$ and $\widetilde{x}_{j}=\left(x_{j}, \alpha_{x_{j}} \%, \beta_{x_{j}} \%\right), j=1,2, \ldots, n$ be non-negative $P L R$ fuzzy numbers as well as $\alpha_{x_{j}} \in\left[0, s_{j}\right]$ and $\beta_{x_{j}} \in\left[0, r_{j}\right]$ also

$$
\widetilde{Z}=(\widehat{z}, \widehat{\alpha} \%, \widehat{\beta} \%)=\sum_{j=1}^{n} \widetilde{c}_{j} \otimes \widetilde{x}_{j}
$$

is the objective function of fully fuzzy linear programming problem (5) then

$$
\begin{aligned}
& \min _{j=1,2, \ldots, n}\left\{\alpha_{c_{j}}\right\} \leq \widehat{\alpha} \leq \max _{j=1,2, \ldots, n}\left\{s_{j}+\alpha_{c_{j}}\right\} \\
& \min _{j=1,2, \ldots, n}\left\{\beta_{c_{j}}\right\} \leq \widehat{\beta} \leq \max _{j=1,2, \ldots, n}\left\{r_{j}+\beta_{c_{j}}\right\}
\end{aligned}
$$

Proof.

$$
\begin{aligned}
& \forall j=1, \ldots, n, \alpha_{x_{j}} \in\left[0, s_{j}\right] \text { and } \beta_{x_{j}} \in\left[0, r_{j}\right] \Rightarrow 0 \leq \alpha_{x_{j}} \leq s_{j} \quad \text { and } 0 \leq \beta_{x_{j}} \leq r_{j} \\
& \Rightarrow \forall j=1,2, \ldots, n \quad, \quad \alpha_{c_{j}} \leq \alpha_{x_{j}}+\alpha_{c_{j}} \leq s_{j}+\alpha_{c_{j}} \quad, \quad \beta_{c_{j}} \leq \beta_{x_{j}}+\beta_{c_{j}} \leq r_{j}+\beta_{c_{j}}
\end{aligned}
$$

So

$$
\begin{aligned}
\min _{j=1, \ldots, n}\left\{\alpha_{c_{j}}\right\} & \leq \min _{j=1, \ldots, n}\left\{\alpha_{x_{j}}+\alpha_{c_{j}}\right\} \\
\max _{j=1, \ldots, n}\left\{\alpha_{x_{j}}+\alpha_{c_{j}}\right\} & \leq \max _{j=1, \ldots, n}\left\{s_{j}+\alpha_{c_{j}}\right\} \\
\min _{j=1, \ldots, n}\left\{\beta_{c_{j}}\right\} & \leq \min \left\{\beta_{x_{j}}+\beta_{c_{j}}\right\} \\
\max _{j=1, \ldots, n}\left\{\beta_{x_{j}}+\beta_{c_{j}}\right\} & \leq \max _{j=1, \ldots, n}\left\{r_{j}+\beta_{c_{j}}\right\}
\end{aligned}
$$

According to Theorem 1 and above inequalities we obtain

$$
\begin{array}{r}
\min _{j=1,2, \ldots, n}\left\{\alpha_{c_{j}}\right\} \leq \widehat{\alpha} \leq \max _{j=1,2, \ldots, n}\left\{s_{j}+\alpha_{c_{j}}\right\} \\
\min _{j=1,2, \ldots, n}\left\{\beta_{c_{j}}\right\} \leq \widehat{\beta} \leq \max _{j=1,2, \ldots, n}\left\{r_{j}+\beta_{c_{j}}\right\}
\end{array}
$$

Example 3. Consider the following fully fuzzy linear programming problem:

$$
\begin{array}{cl}
\text { Min } \quad \widetilde{Z}= & \widetilde{c}_{1} \otimes \widetilde{x}_{1} \oplus \widetilde{c}_{2} \otimes \widetilde{x}_{2} \\
\text { s.t. } & \widetilde{a}_{11} \otimes \widetilde{x}_{1} \oplus \widetilde{a}_{12} \otimes \widetilde{x}_{2} \succeq \widetilde{b}_{1} \\
& \widetilde{a}_{21} \otimes \widetilde{x}_{1} \oplus \widetilde{a}_{22} \otimes \widetilde{x}_{2} \preceq \widetilde{b}_{2} \\
& \widetilde{x}_{1}, \widetilde{x}_{2} \succeq 0
\end{array}
$$

where $\widetilde{c}_{1}=(12,6 \%, 4 \%), \quad \widetilde{c}_{2}=(20,5 \%, 5 \%), \quad \widetilde{a}_{11}=(8,5 \%, 4 \%), \quad \widetilde{a}_{12}=(10,5 \%, 4 \%), \quad \widetilde{a}_{21}=(15,10 \%, 8 \%)$, $\widetilde{a}_{22}=(18,6 \%, 5 \%), \widetilde{b}_{1}=(60,10 \%, 6 \%), \widetilde{b}_{2}=(120,6 \%, 8 \%), \widetilde{x}_{1}=\left(x_{1}, \alpha_{x_{1}} \%, \beta_{x_{1}} \%\right)$ and $\widetilde{x}_{2}=\left(x_{2}, \alpha_{x_{2}} \%, \beta_{x_{2}} \%\right)$ be PLR fuzzy numbers. An addition maximum variation range of $\widetilde{x}_{1}$ and $\widetilde{x}_{2}$ are predetermined in Table 1.

Maximum variation range of $\widetilde{x}_{1}$ and $\widetilde{x}_{2}$

\begin{tabular}{lcclcc}
\hline & \multicolumn{2}{c}{$\widetilde{x}_{1}$} & & \multicolumn{2}{c}{$\widetilde{x}_{2}$} \\
\cline { 2 - 3 } \cline { 5 - 6 } & $s_{1}$ & $r_{1}$ & & $s_{2}$ & $r_{2}$ \\
\hline Maximum variation range & $6 \%$ & $4 \%$ & & $3 \%$ & $4 \%$ \\
\hline
\end{tabular}


Therefor

$$
\begin{aligned}
0 \leq \alpha_{x_{1}} \leq s_{1} & \Rightarrow \alpha_{c_{1}} \leq \alpha_{x_{1}}+\alpha_{c_{1}} \leq s_{1}+\alpha_{c_{1}} \\
0 \leq \alpha_{x_{2}} \leq s_{2} & \Rightarrow 6 \leq \alpha_{x_{1}}+\alpha_{c_{1}} \leq s_{1}+6=6+6=12 \\
& \Rightarrow 5 \leq \alpha_{c_{2}} \leq \alpha_{x_{2}}+\alpha_{c_{2}} \leq s_{2}+\alpha_{c_{2}} \\
0 \leq \beta_{x_{1}} \leq r_{1} & \Rightarrow \beta_{c_{1}} \leq \beta_{x_{1}}+\beta_{c_{1}} \leq r_{1}+\beta_{c_{1}} \\
& \Rightarrow 4 \leq \beta_{x_{1}}+\beta_{c_{1}} \leq r_{1}+4=4+8=8 \\
0 \leq \beta_{x_{2}} \leq r_{2} & \Rightarrow \beta_{c_{2}} \leq \beta_{x_{2}}+\beta_{c_{2}} \leq r_{2}+\beta_{c_{2}} \\
& \Rightarrow 5 \leq \beta_{x_{2}}+\beta_{c_{2}} \leq r_{2}+5=4+5=9
\end{aligned}
$$

Thus

$$
\begin{aligned}
& \min \left\{\alpha_{c_{1}}+\alpha_{x_{1}}, \alpha_{c_{2}}+\alpha_{x_{2}}\right\} \geq \min \{6,5\}=5 \\
& \max \left\{\alpha_{c_{1}}+\alpha_{x_{1}}, \alpha_{c_{2}}+\alpha_{x_{2}}\right\} \leq \max \{12,8\}=12
\end{aligned}
$$

and

$$
\begin{aligned}
& \min \left\{\beta_{c_{1}}+\beta_{x_{1}}, \beta_{c_{2}}+\beta_{x_{2}}\right\} \geq \min \{4,5\}=4 \\
& \max \left\{\beta_{c_{1}}+\beta_{x_{1}}, \beta_{c_{2}}+\beta_{x_{2}}\right\} \leq \max \{8,9\}=9
\end{aligned}
$$

According to Theorem 1 we find

$$
5 \leq \widehat{\alpha} \leq 12, \quad 4 \leq \widehat{\beta} \leq 9
$$

where $\widetilde{x}_{1}$ and $\widetilde{x}_{2}$ are feasible solutions and $\widetilde{Z}=(\widehat{z}, \widehat{\alpha} \%, \widehat{\beta} \%)$ is a value of objective function proportional to $\widetilde{x}_{1}$ and $\widetilde{x}_{2}$. The variation range of $\widehat{\alpha}$ and $\widehat{\beta}$ indicate that, for each fuzzy feasible solutions, $\widehat{\alpha}$ vary between $5 \%$ and $12 \%$ and $\widehat{\beta}$ vary between $4 \%$ and $9 \%$ (See Figure 1 ). Especially, if $\widetilde{x}_{1}^{*}$ and $\widetilde{x}_{2}^{*}$ are optimal feasible solutions then variation range of $\widetilde{Z}^{*}$ can be predicted as follows:

$$
\widetilde{Z}^{*}=\left\{\left(z^{*}, \alpha^{*} \%, \beta^{*} \%\right) \mid \alpha^{*} \in[5,12], \beta^{*} \in[4,9]\right\}
$$

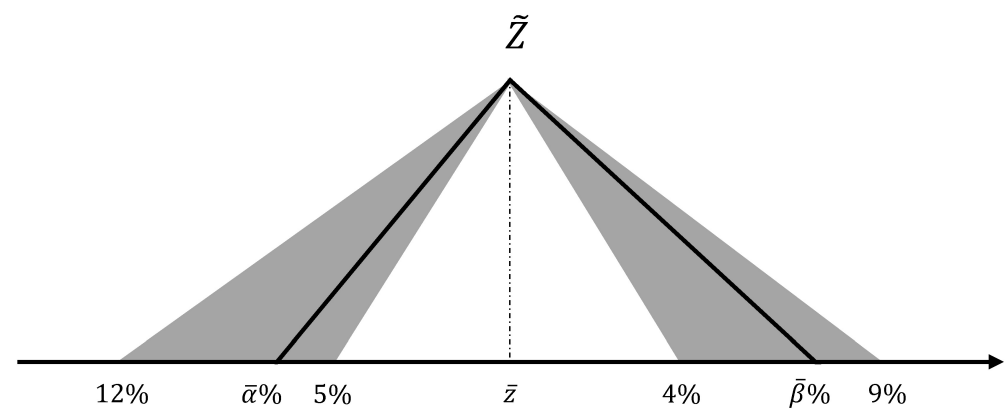

Figure 1. The variation range of left and right side of $\widetilde{Z}$

Note that Theorem 2 imply that the maximum variation range of left and right side of $\widetilde{Z}^{*}$ may be changed if the maximum variation range of left and right side of fuzzy variable is changed.

In other words:

- The minimum variation range of left and right side of $\widetilde{Z}^{*}$ is independent of fuzzy variables' variation range.

- The maximum variation range of left and right side of $\widetilde{Z}^{*}$ is dependent on fuzzy variables' variation range.

\section{Predicting optimal value of objective function}

Here, we describe how the optimal value of objective function in fully fuzzy linear programming problems are predictable when all of the parameters and variables are symmetric triangular fuzzy numbers. 
Theorem 3. If $\widetilde{A}=(a, \alpha \%)$ and $\widetilde{B}=(b, \beta \%)$ are symmetric $P L R$ fuzzy number and $a+b \neq 0$, then $\widetilde{A} \oplus \widetilde{B}$ is symmetric $P L R$ fuzzy number.

Proof. $\widetilde{A}=(a, \alpha \%)$ and $\widetilde{B}=(b, \beta \%)$ are symmetric $P L R$ fuzzy number, then we can show $\widetilde{A}=(a, \alpha \%, \alpha \%)$ and $\widetilde{B}=(b, \beta \%, \beta \%)$ and we have

$$
\widetilde{A} \oplus \widetilde{B}=\left(a+b, \frac{\alpha|a|+\beta|b|}{|a+b|} \%, \frac{\alpha|a|+\beta|b|}{|a+b|} \%\right) .
$$

Shows that $\widetilde{A} \oplus \widetilde{B}$ is symmetric $P L R$ fuzzy number.

Theorem 4. If $\widetilde{A}=(a, \alpha \%)$ and $\widetilde{B}=(b, \beta \%)$ are symmetric $P L R$ fuzzy number and $\widetilde{A}, \widetilde{B} \not 0$, then $\widetilde{A} \otimes \widetilde{B}$ is symmetric $P L R$ fuzzy number.

Proof. Analogous to the proof of Theorem 3.

Lemma 1. Let $\widetilde{A}_{j}=\left(a_{j}, \alpha_{j} \%\right)$ and $\widetilde{B}_{j}=\left(b_{j}, \beta_{j} \%\right), j=1,2, \ldots, n$ be non-negative symmetric $P L R$ fuzzy numbers and

$$
\widetilde{Z}=\sum_{j=1}^{n} \widetilde{A}_{j} \otimes \widetilde{B}_{j}
$$

then $\widetilde{Z}$ is symmetric $P L R$ fuzzy numbers.

Lemma 2. If $\widetilde{A}=(a, \alpha \%)$ is symmetric triangular fuzzy number then $\mathfrak{R}(\widetilde{A})=a$.

Suppose all the parameters and variables in the fully fuzzy linear programming (5) are triangular symmetric fuzzy numbers, then as a consequence of Lemma 1 the result of $\sum_{j=1}^{n} \widetilde{c}_{j} \otimes \widetilde{x}_{j}$ and $\sum_{j=1}^{n} \widetilde{a}_{i j} \otimes \widetilde{x}_{j}$ are symmetric triangular fuzzy numbers. In addition

$$
\begin{array}{ll}
\mathfrak{R}\left(\sum_{j=1}^{n} \widetilde{c}_{j} \otimes \widetilde{x}_{j}\right)=\sum_{j=1}^{n} c_{j} x_{j} & \\
\mathfrak{R}\left(\sum_{j=1}^{n} \widetilde{a}_{i j} \otimes \widetilde{x}_{j}\right)=\sum_{j=1}^{n} a_{i j} x_{j}, & i=1,2, \ldots, m \\
\mathfrak{R}\left(\widetilde{b}_{i}\right)=b_{i}, & i=1,2, \ldots, m \\
\mathfrak{R}\left(\widetilde{x}_{j}\right)=x_{j}, & j=1,2, \ldots, n
\end{array}
$$

In fact, the left and right sides of $P L R$ fuzzy parameters and variables are ignored by applying defuzzification. Hence the fully fuzzy linear programming (5) with using ranking function is converted to crisp linear programming as follows:

$$
\begin{aligned}
& \underset{(\operatorname{Max})}{\operatorname{Min}} \quad Z=\sum_{j=1}^{n} c_{j} x_{j} \\
& \text { s.t. } \quad \sum_{j=1}^{n} a_{i j} x_{j} \stackrel{\geq}{=} b_{i} \quad, \quad i=1,2, \ldots, m \\
& x_{j} \geq 0
\end{aligned}
$$

Assume that $x_{j}^{*}, j=1,2, \ldots, n$ are optimal feasible solutions of linear programming $(6)$ then $\widetilde{Z^{*}}=\left(z^{*}, \alpha^{*} \%\right)$ is a prediction for the optimal objective function of model (5) where

$$
z^{*}=c_{1} x_{1}^{*}+c_{2} x_{2}^{*}+\cdots+c_{n} x_{n}^{*}
$$

and

$$
\min _{j=1, \ldots, n}\left\{\alpha_{c_{j}}\right\} \leq \alpha^{*} \leq \max _{j=1, \ldots, n}\left\{\alpha_{c_{j}}+s_{j}\right\} .
$$

Example 4. A company produces 3 kinds of goods with high profitability. Market estimation shows that each of these products can be sold at $\$ 865, \$ 580$ and $\$ 470$ with maximum variation rate of $9 \%, 7.5 \%$ and $12 \%$ 
respectively. The company has 3 stages of production and each of these products require a proportion of the capacity of these stages. Maximum capacity of each production stages and proportion of the needed capacity for each product with its' maximum variation rate are given in Table 2.

Table 2

Require a portion of the capacity of production stages for each products

\begin{tabular}{|c|c|c|c|c|c|c|}
\hline \multirow[b]{2}{*}{ Products } & \multicolumn{2}{|c|}{ Production Stage 1} & \multicolumn{2}{|c|}{ Production Stage 2} & \multicolumn{2}{|c|}{ Production Stage 3} \\
\hline & Value & $\alpha$ & Value & $\alpha$ & Value & $\alpha$ \\
\hline P1 & 1 & $0 \%$ & 5 & $3 \%$ & 3 & $1 \%$ \\
\hline P2 & 0.8 & $0 \%$ & 3 & $5 \%$ & 2 & $2 \%$ \\
\hline P3 & 2 & $0 \%$ & 2.5 & $4 \%$ & 1.2 & $4 \%$ \\
\hline Maximum Capacity & 200 & $2 \%$ & 680 & $6 \%$ & 400 & $3 \%$ \\
\hline
\end{tabular}

$\alpha$ : Maximum variation rate of each data

Since these goods are all best-selling, the company wants to get maximum production capacity. Here the manager really wants to know the amount of maximum profit and variation rate of profit when maximum variation rate of products is predetermined to be $5 \%$ and $10 \%$ respectively. This problem of goods production in the way that profit is maximized and the restrictions are met, is formulated as the following fully fuzzy linear programming.

$$
\begin{aligned}
\operatorname{Max} \widetilde{Z}= & (865,9 \%) \otimes \widetilde{x}_{1} \oplus(580,7.5 \%) \otimes \widetilde{x}_{2} \oplus(470,12 \%) \otimes \widetilde{x}_{3} \\
\text { s.t. } \quad & (1,0 \%) \otimes \widetilde{x}_{1} \oplus(0.8,0 \%) \otimes \widetilde{x}_{2} \oplus(2,0 \%) \otimes \widetilde{x}_{3} \preceq(200,2 \%) \\
& (5,3 \%) \otimes \widetilde{x}_{1} \oplus(3,5 \%) \otimes \widetilde{x}_{2} \oplus(2.5,4 \%) \otimes \widetilde{x}_{3} \preceq(680,6 \%) \\
& (3,1 \%) \otimes \widetilde{x}_{1} \oplus(2,2 \%) \otimes \widetilde{x}_{2} \oplus(1.2,4 \%) \otimes \widetilde{x}_{3} \preceq(400,3 \%) \\
& \widetilde{x}_{1}, \widetilde{x}_{2}, \widetilde{x}_{3} \succeq 0
\end{aligned}
$$

By appealing to the ranking function for defuzzification, the above fully fuzzy linear programming is converted to simple crisp linear programming as shown by the following

$$
\begin{array}{cr}
\operatorname{Max} \quad Z= & 865 x_{1}+580 x_{2}+470 x_{3} \\
\text { s.t. } & x_{1}+0.8 x_{2}+2 x_{3} \leq 200 \\
& 5 x_{1}+3 x_{2}+2.5 x_{3} \leq 680 \\
& 3 x_{1}+2 x_{2}+1.2 x_{3} \leq 400 \\
& x_{1}, x_{2}, x_{3} \geq 0
\end{array}
$$

\begin{tabular}{|c|c|c|c|c|c|}
\hline & \multicolumn{3}{|c|}{ Maximum variation range of products } & \multirow{2}{*}{$\begin{array}{l}\text { Maximum variation } \\
\text { range of profit }\end{array}$} & \multirow[t]{2}{*}{$\widetilde{Z}^{*}$} \\
\hline & $\mathrm{P} 1\left(x_{1}\right)$ & $\mathrm{P} 2\left(x_{2}\right)$ & P3 $\left(x_{3}\right)$ & & \\
\hline 1 & $5 \%$ & $5 \%$ & $5 \%$ & $17 \%$ & $(2520,17 \%)$ \\
\hline 2 & $10 \%$ & $10 \%$ & $10 \%$ & $22 \%$ & $(2520,22 \%)$ \\
\hline
\end{tabular}

The maximum value of objective function of linear programming problem (8) is ( $\left.Z^{*}\right)$ equal to 2520 . Since fully fuzzy linear programming problem (7) is simplified to crisp linear programming problem $(8), Z^{*}=2520$ is an appropriate approximation for optimal profit irrespective of variation range of fuzzy parameters and fuzzy variables. Then, by applying Theorem 2 , according to maximum variation range of products, maximum variation rate of optimal profit is calculated, as given in Table 3.

Maximum variation range of products and profit for Example 4

\section{Conclusion}

The given approach of in this paper differs significantly from other similar research studies which were conducted in the class of fuzzy linear programming problems. Here we introduced the PLR fuzzy numbers which are applicable to many branches of knowledge. In addition, in contrast to arithmetic operations on $L R$ fuzzy numbers which are usually very complicated and voluminous, we have developed $P L R$ fuzzy numbers with simpler multiplication operations and fewer computational procedures. Therefor in this method we are 
able to reduce the lengthy numerical algorithms required in an arithmetic operations on fuzzy numbers when the number of multiplication operations are more than the number of additional operations. Furthermore, by applying our percentage form of fuzzy numbers in fuzzy linear programming problem, we are able to predict maximum variation range of optimal value of objective function when the maximum variation range of variables are predetermined.

\section{References}

1 Nehi, H. M., Maleki, H. R., \& Mashinchi, M. (2004). Solving fuzzy number linear programming problem by lexicographic ranking function. Italian Journal of Pure and Applied Mathematics, 15, 9-20.

2 Tanaka, H., Okuda, T., \& Asai, K. (1973). On fuzzy-mathematical programming. Journal of Cybernetics, 3, 37-46.

3 Bellman, R. E., \& Zadeh, L. A. (1970). Decision-making in a fuzzy environment. Management Science, $17,141-164$.

4 Zimmermann, H.-J. (1978). Fuzzy programming and linear programming with several objective functions. Fuzzy Sets and Systems, 1, 45-55.

5 Campos, L., \& Verdegay, J. (1989). Linear programming problems and ranking of fuzzy numbers. Fuzzy Sets and Systems, 32, 1-11.

6 Ebrahimnejad, A., Nasseri, S.H., Lotfi, F. H., \& Soltanifar, M. (2010). A primal-dual method for linear programming problems with fuzzy variables. European Journal of Industrial Engineering, 4, 189-209.

7 Ganesan, K., \& Veeramani, P. (2006). Fuzzy linear programs with trapezoidal fuzzy numbers. Annals of Operations Research, 143, 305-315.

8 Mahdavi-Amiri, N., \& Nasseri, S. (2006). Duality in fuzzy number linear programming by use of a certain linear ranking function. Applied Mathematics and Computation, 180, 206-216.

9 Mahdavi-Amiri, N., \& Nasseri, S. (2007). Duality results and a dual simplex method for linear programming problems with trapezoidal fuzzy variables. Fuzzy Sets and Systems, 158, 1961-1978.

10 Maleki, H., Tata, M., \& Mashinchi, M. (2000). Linear programming with fuzzy variables. Fuzzy Sets and Systems, 109, 21-33.

11 Chen, L.H., \& Ko, W.C. (2009). Fuzzy linear programming models for new product design using QFD with FMEA. Applied Mathematical Modelling, 33, 633-647.

12 Gupta, A., Kumar, A., \& Sharma, M. (2013). Applications of fuzzy linear programming with generalized lr flat fuzzy parameters. Fuzzy Information and Engineering, 5, 475-492.

13 Ebrahimnejad, A., \& Tavana, M. (2014). A novel method for solving linear programming problems with symmetric trapezoidal fuzzy numbers. Applied Mathematical Modelling, 38, 4388-4395.

14 Dong, J.-y., \& Wan, S.-P. (2018). A new trapezoidal fuzzy linear programming method considering the acceptance degree of fuzzy constraints violated. Knowledge-Based Systems, 148, 100-114.

15 Allahviranloo, T., Lotfi, F. H., Kiasary, M. K., Kiani, N. A., \& Alizadeh, L. (2008). Solving fully fuzzy linear programming problem by the ranking functhion. Applied Matematical Sciences, 2, 19-32.

16 Buckley, J. J., \& Feuring, T. (2000). Evolutionary algorithm solution to fuzzy problems: Fuzzy linear programming. Fuzzy Sets and Systems, 109, 35-53.

17 Hashemi, S. M., Modarres, M., Nasrabadi, E., \& Nasrabadi, M. M. (2006). Fully fuzzified linear programming, solution and duality. J. Intell. Fuzzy Syst., 17, 253-261.

18 Kumar, A., Kaur, J., \& Singh, P. (2010). Fuzzy optimal solution of fully fuzzy linear programming problems with inequality constraints. International Journal of Mathematical and Computer Sciences, 6, $37-41$.

19 Kumar, A., Kaur, J., \& Singh, P. (2011). A new method for solving fully fuzzy linear programming problems. Applied Mathematical Modelling, 35, 817-823.

20 Lotfi, F. H., Allahviranloo, T., Jondabeh, M. A., \& Alizadeh, L. (2009). Solving a full fuzzy linear programming using lexicography method and fuzzy approximate solution. Applied Mathematical Modelling, 33, 3151-3156.

21 Kaur, J., \& Kumar, A. (2013). Mehar's method for solving fully fuzzy linear programming problems with L-R fuzzy parameters. Applied Mathematical Modelling, 37, 7142-7153. 
22 Cheng, H., Huang, W., \& Cai, J. (2013). Method for solving fully fuzzy linear programming problems using deviation degree measure. Journal of Systems Engineering and Electronics, 24, 793-799.

23 Chehlabi, M., \& Allahviranloo, T. (2018). Positive or negative solutions to first-order fully fuzzy linear differential equations under generalized differentiability. Applied Soft Computing, 70, 359-370.

24 Najafi, H. S., Edalatpanah, S., \& Dutta, H. (2016). A nonlinear model for fully fuzzy linear programming with fully unrestricted variables and parameters. Alexandria Engineering Journal, 55, 2589-2595.

25 Dubois, D., \& Prade, H. (1980). Systems of linear fuzzy constraints. Fuzzy Sets and Systems, 3, 37-48.

26 Yager, R. R. (1981). A procedure for ordering fuzzy subsets of the unit interval. Information Sciences, 24, 143-161.

\author{
С.М. Давуди, Н.А. Абдул Рахман
}

\title{
Анықемес сызықтық бағдарламалау есебіндегі оңтайлы шешімді болжау
}

\begin{abstract}
Мақалада анықемес сандардың пайдалы түрі мен оның арифметикасы болып табылатын, анықемес сандардың LR пайыздық түрін анықтауға тырысқан. Авторлар анықемес сандардың осы формасын қолдана отырып, анықемес объективті функцияның оңтайлы мәнінің өзгеруінің максималды диапазонын қалай болжауға болатындығын көрсеткен. Анықемес мәселелер әдетте күрделі түрде шешілетіндіктен, бұл зерттеудің мақсаты - менеджер мәселелерді шешпестен оңтайлы шешім болжамына $\left(Z^{*}\right)$ қол жеткізе алатындай етіп түпкілікті шешім үшін болжам түрін шығару болып табылады. Соңында, анықемес сызықтық бағдарламалау кезінде анықемес объективті функцияның оңтайлы мәні анықемес айнымалының өзгеруінің максималды диапазоны алдын-ала анықталған кезде болжанады.
\end{abstract}

Kiлm сөздер: анықемес сандардың пайыздық түрі, тақ сан, анықемес сызықтық бағдарламалау, анықемес арифметика.

\author{
C.M. Давуди, Н.А. Абдул Рахман
}

\section{Прогнозирование оптимального решения в задаче нечеткого линейного программирования}

В статье предпринята попытка определить процентную форму LR нечетких чисел, которая является полезной формой нечетких чисел и ее арифметикой. Авторы показывают, как можно предсказать максимальный диапазон изменения оптимального значения нечеткой целевой функции с помощью этой формы нечетких чисел. Поскольку нечеткие проблемы, как правило, решаются сложным образом, цель этого исследования - выпустить своего рода прогноз для окончательного решения таким образом, чтобы менеджер мог получить доступ к прогнозу оптимального решения $\left(Z^{*}\right)$, не решая проблемы. Наконец, оптимальное значение нечеткой целевой функции при нечетком линейном программировании предсказывается, когда предопределен максимальный диапазон изменения нечеткой переменной.

Ключевые слова: процентная форма нечетких чисел, нечеткое число, нечеткое линейное программирование, нечеткая арифметика. 